\title{
Development for a supercompact $X$-band pulse compression system and its application at SLAC
}

\author{
Juwen W. Wang, Sami G. Tantawi, Chen Xu, Matt Franzi, Patrick Krejcik, Gordon Bowden, \\ Shantha Condamoor, Yuantao Ding, Valery Dolgashev, John Eichner, Andrew Haase, \\ James R. Lewandowski, and Liling Xiao \\ SLAC National Accelerator Laboratory, Menlo Park, California 94025, USA
}

(Received 28 June 2017; published 9 November 2017)

\begin{abstract}
We have successfully designed, fabricated, installed, and tested a super compact $X$-band SLAC Energy Doubler system at SLAC. It is composed of an elegant $3 \mathrm{~dB}$ coupler-mode converter-polarizer coupled to a single spherical energy storage cavity with high $Q_{0}$ of 94000 and a diameter less than $12 \mathrm{~cm}$. The available rf peak power of $50 \mathrm{MW}$ can be compressed to a peak average power of more than $200 \mathrm{MW}$ in order to double the kick for the electron bunches in a rf transverse deflector system and greatly improve the measurement resolution of both the electron bunches and the x-ray free-electron laser pulses. The design physics and fabrication as well as the measurement results will be presented in detail. High-power operation has demonstrated the excellent performance of this rf compression system without rf breakdown, sign of pulse heating, and rf radiation.
\end{abstract}

DOI: 10.1103/PhysRevAccelBeams.20.110401

\section{INTRODUCTION}

A diagnostics system for $\mathrm{x}$-ray temporal measurement has been intensively used at the Linac Coherent Light Source (LCLS) operation. This system is based on two $X$-band traveling wave rf transverse deflectors, a magnetic spectrometer, and the Ce:YAG screen located downstream of the free-electron laser (FEL) undulator [1]. Its layout is shown in Fig. 1. The measured resolution was below $1 \mathrm{fs}$ rms at soft $\mathrm{x}$-ray energies and $4 \mathrm{fs}$ rms at hard $\mathrm{x}$-ray energies. A pulse duration of less than 3 fs FWHM at soft x-ray energy has been measured, and the electron trapping in X-ray FELs was experimentally observed in the deep-saturation regime. In order to further improve the temporal resolution, we increased the deflecting strength by upgrading the existing power source with rf pulse compression technology. After the installation of the new SLAC Energy Doubler (SLED) system, the measured resolution was below $0.5 \mathrm{fs}$ rms at soft $\mathrm{x}$-ray energies and $2 \mathrm{fs}$ rms at hard x-ray energies.

In recent years, we have studied, designed, and fabricated a novel supercompact SLED system to double the peak deflection [2]. This paper describes its principle, design and manufacture related issues, microwave measurement, and high-power operation results as well as other technical advances in detail.

\footnotetext{
jywap@slac.stanford.edu
}

Published by the American Physical Society under the terms of the Creative Commons Attribution 4.0 International license. Further distribution of this work must maintain attribution to the author(s) and the published article's title, journal citation, and DOI.

\section{DESIGN OF SUPERCOMPACT SLED SYSTEM}

More than 40 years ago, SLAC developed the SLED system to obtain high peak rf power in exchange for a reduction in the rf pulse length by a passive technique called "pulse compression" [3]. As shown in Fig. 2, the first part of a klystron pulse was stored in two low-loss cylindrical tuned cavities installed downstream of the klystron. For the remaining part of the pulse (usually to be the filling time of the feeding accelerator structure), the phase of the klystron was reversed by 180 electrical degrees, and the sum of the power now being discharged by the cavities plus the direct klystron power resulted in a net power gain. The key components of a SLED system include a $3 \mathrm{db}$ coupler with two $90^{\circ}$ apart divided power ports and two high-Q energy storage cavities.

There are two major innovative changes in our new compact SLED system presented here: the traditional $3 \mathrm{~dB}$ coupler will be replaced by a more compact dual-mode circular polarizer, and two cylindrical energy storage cavities will be replace by a high- $Q$ spherical cavity with two polarized modes.

The LCLS deflector is made of two traveling structures; each one is a $1.0 \mathrm{~m}$ long, constant impedance structure with a transverse impedance of $41.9 \mathrm{M} \Omega / \mathrm{m}$, filling time $\mathrm{T}_{f}=$ $106 \mathrm{~ns}$ (group velocity of $3.165 \%$ speed of light), and attenuation factor $\tau=0.62 \mathrm{~Np}$. If we assume the similar average rf power of $106 \mathrm{~ns}$ pulses for both the SLEDed pulse and non-SLEDed flat pulses with similar average power to feed a backward wave constant impedance deflector, the corresponding kick voltages are shown in Fig. 3.

We need to optimize the SLED system by calculating its total gain for various coupling coefficients for the cavity $Q_{0}$ 


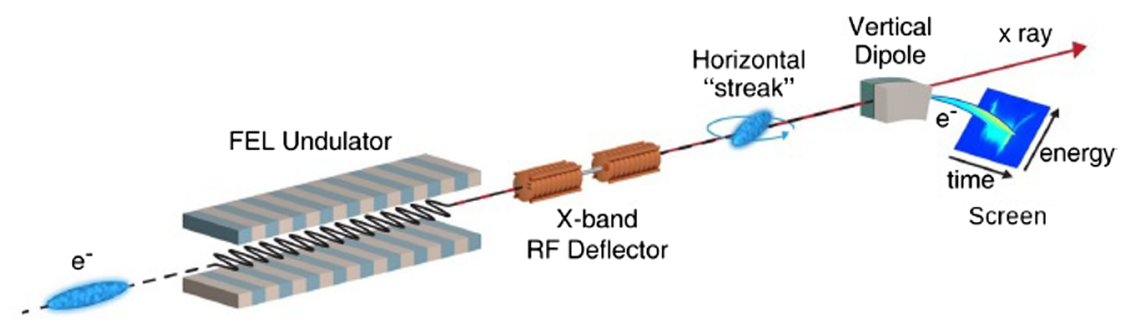

FIG. 1. Diagnostics layout of the x-ray temporal measurement at the LCLS.

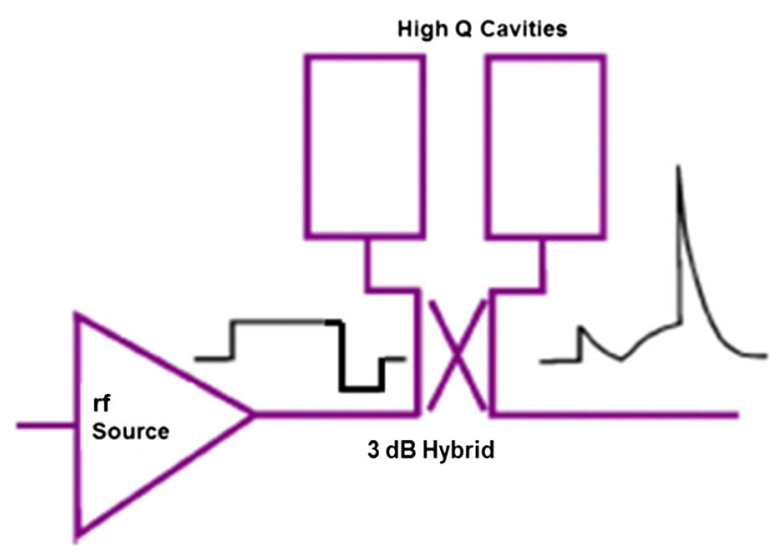

FIG. 2. Working scheme for a basic SLED system.

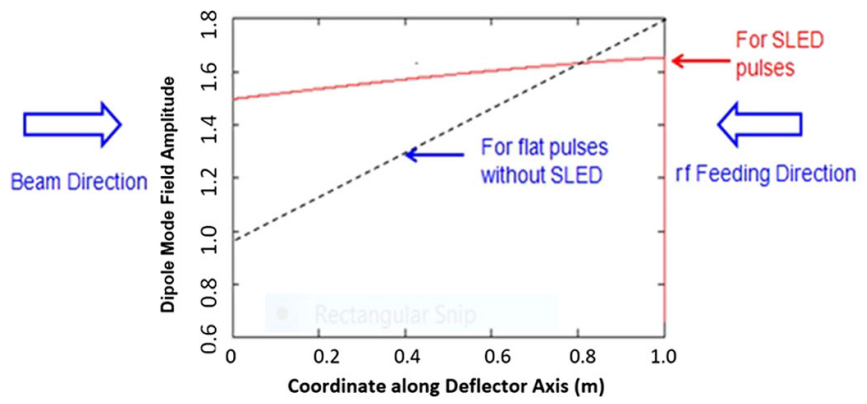

FIG. 3. Kick voltage along the deflector structure.

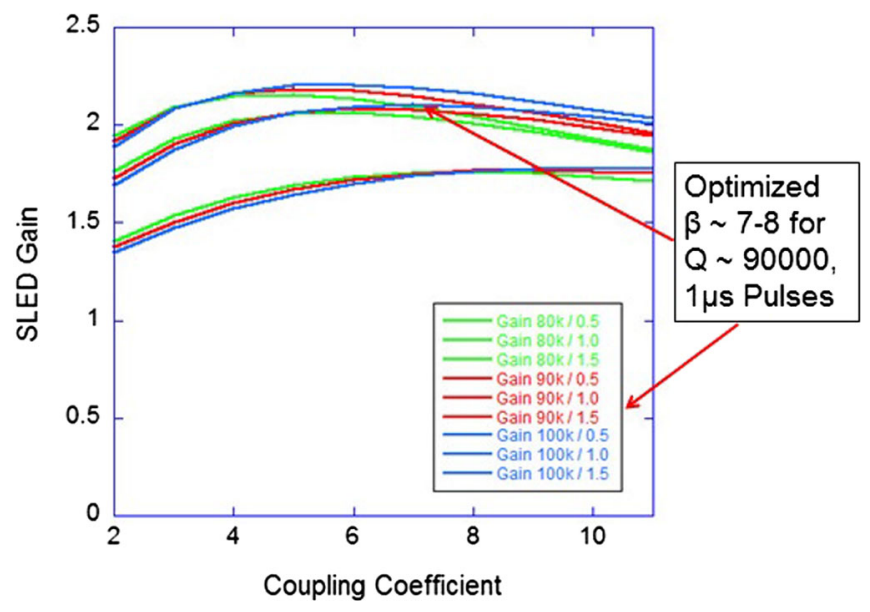

FIG. 4. SLED gain as a function of the coupling coefficient for various pulse widths and storage cavity $Q_{0}$ values. value and pulse length. Figure 4 shows that the peak gain larger than a factor of 2 can be obtained for $Q_{0} \sim 90000$ and $1 \mu$ s pulses if the overcoupling coefficient $\beta$ is optimized to be 7-8 for $11424 \mathrm{MHz}$.

\section{A. Unified $3 \mathrm{~dB}$ coupler-mode convertor-polarizer}

Having all the basic functions of a $3 \mathrm{~dB}$ coupler, a much more compact dual-mode circular polarizer was developed to transform the $\mathrm{TE}_{01}$ mode from a rectangular waveguide into two polarized $\mathrm{TE}_{11}$ modes in quadrature in a circular waveguide as shown in Fig. 5. A detailed description for design procedures can be found in Ref. [4].

The input $\mathrm{TE}_{01}$ mode converts to both $\mathrm{TE}_{01}$ and $\mathrm{TE}_{02}$ modes in a widened rectangular waveguide region. Their magnetic field components will couple to two perpendicular polarized $\mathrm{TE}_{11}$ modes in the circular waveguide. We adjusted geometries so the phases of these two polarized modes are in quadrature separated by $90^{\circ}$.

The circular waveguide feeds a single spherical cavity as shown in Fig. 6. Two polarized sphere modes stored in this spherical cavity will act in a similar fashion and make it much more compact than the original SLED system, which used two separate cylinders cavities.

\section{B. High Q spherical cavity}

The electrical vector potential $F$ for all modes in a sphere with radius $r=a$ can be described by spherical harmonics:

$$
\left(F_{r}\right)_{m n p}=\hat{J}_{n}\left(u_{n p} \frac{r}{a}\right) P_{n}^{m}(\cos \vartheta)\left\{\begin{array}{c}
\cos m \varphi \\
\sin m \varphi
\end{array}\right\} \text {, }
$$

where $\hat{J}_{n}$ is the spherical Bessel Function and $P_{n}^{m}$ are the associated Legendre polynomials with $m \leq n$ [5].

For TE modes, $E_{\varphi}=H_{\theta}=0$ at surface $r=a$. This means $\hat{J}_{n}\left(u_{n p}\right)=0$. Therefore, the sphere radius can be calculated using wave propagation constant $k$ and value of $u_{n p}$ :

$$
a=\frac{u_{n p}}{k}=\frac{c \times u_{n p}}{k}=0.41767 u_{n p}(\mathrm{~cm}) .
$$

The sphere radius is independent of mode index $m$. There are numerous degenerative modes with the same $m$.

In our case, we have chosen $\mathrm{TE}_{m 14}$ modes. There are three possible modes: 


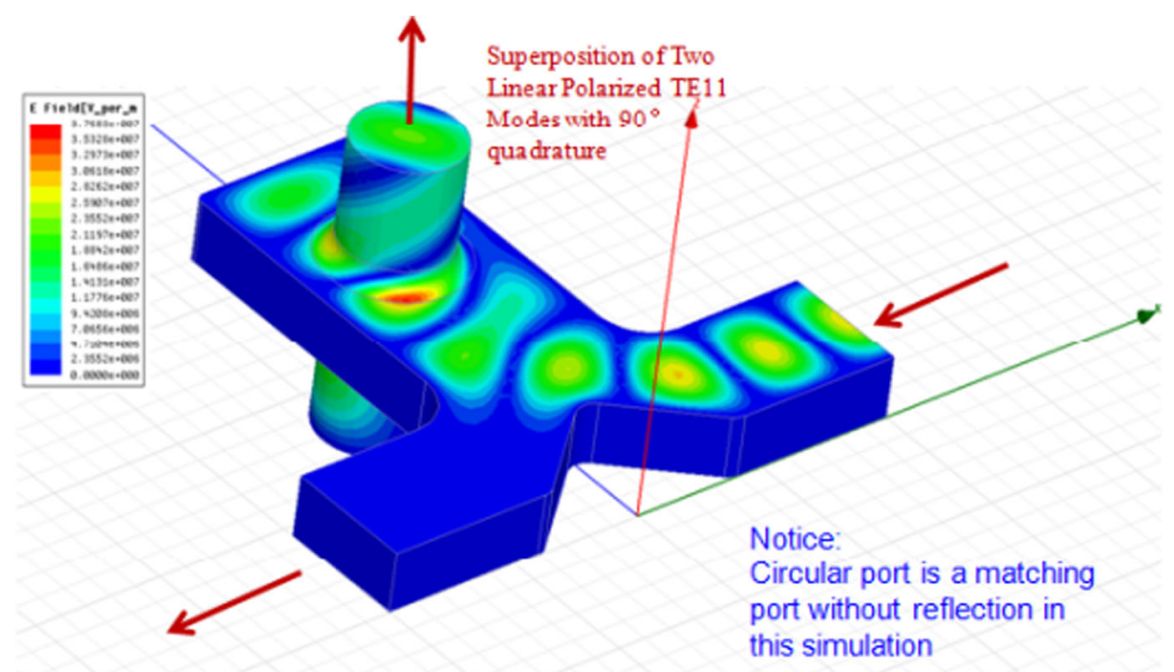

FIG. 5. Schematic view of the dual-mode polarizer.

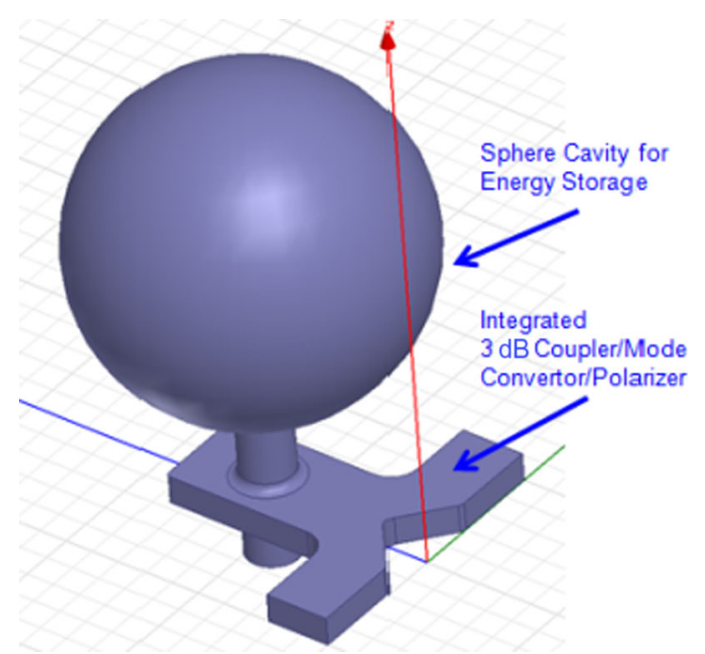

FIG. 6. Construction of the new SLED system.

$$
\begin{gathered}
\left(F_{r}\right)_{014}=\hat{J}_{1}\left(14.066 \frac{r}{a}\right) \cos \vartheta \\
\left(F_{r}\right)_{114}=\hat{J}_{1}\left(14.066 \frac{r}{a}\right) \sin \vartheta \cos \varphi, \\
\left(F_{r}\right)_{114}=\hat{J}_{1}\left(14.066 \frac{r}{a}\right) \sin \vartheta \sin \varphi .
\end{gathered}
$$

For a perfect spherical cavity, these three modes have the same radial mode patterns except that they are rotated $90^{\circ}$ in space from each other, which can be seen in Fig. 6. In reality, they can be slightly distinguished by frequency due to the perturbation from the coupler port. The mode is higher in frequency and very weakly undercoupled due to the feed orientation.

Another interesting property is the sole dependence of $Q_{0}$ on the sphere radius without depending on the mode
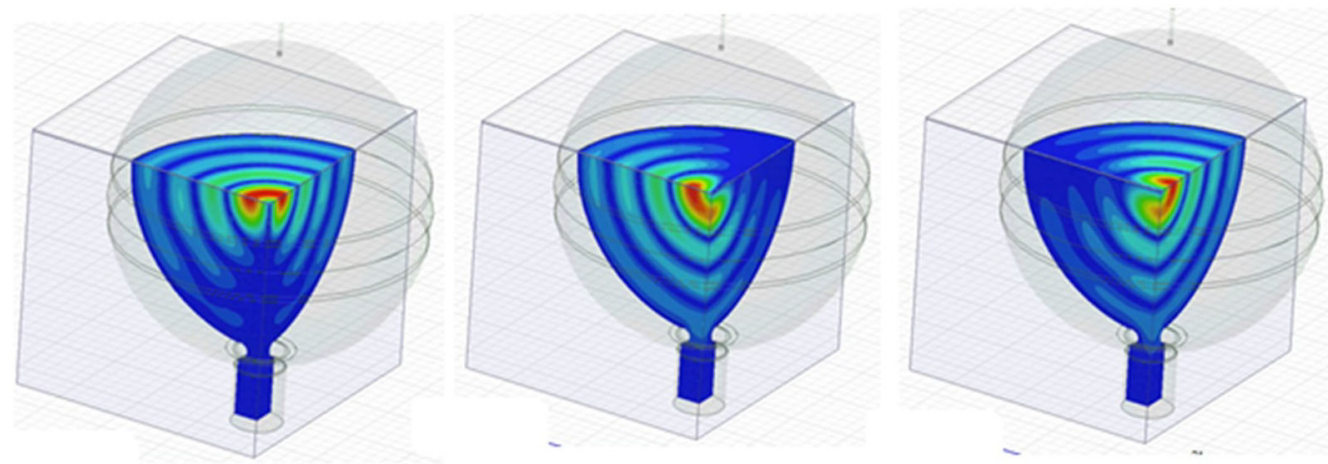

FIG. 7. Two $\mathrm{TE}_{114}$ modes in the middle and right are differently polarized, but with the same coupling to the cylindrical waveguide, and one $\mathrm{TE}_{014}$ mode in the left has different polarization and very weak coupling to the cylindrical waveguide. 


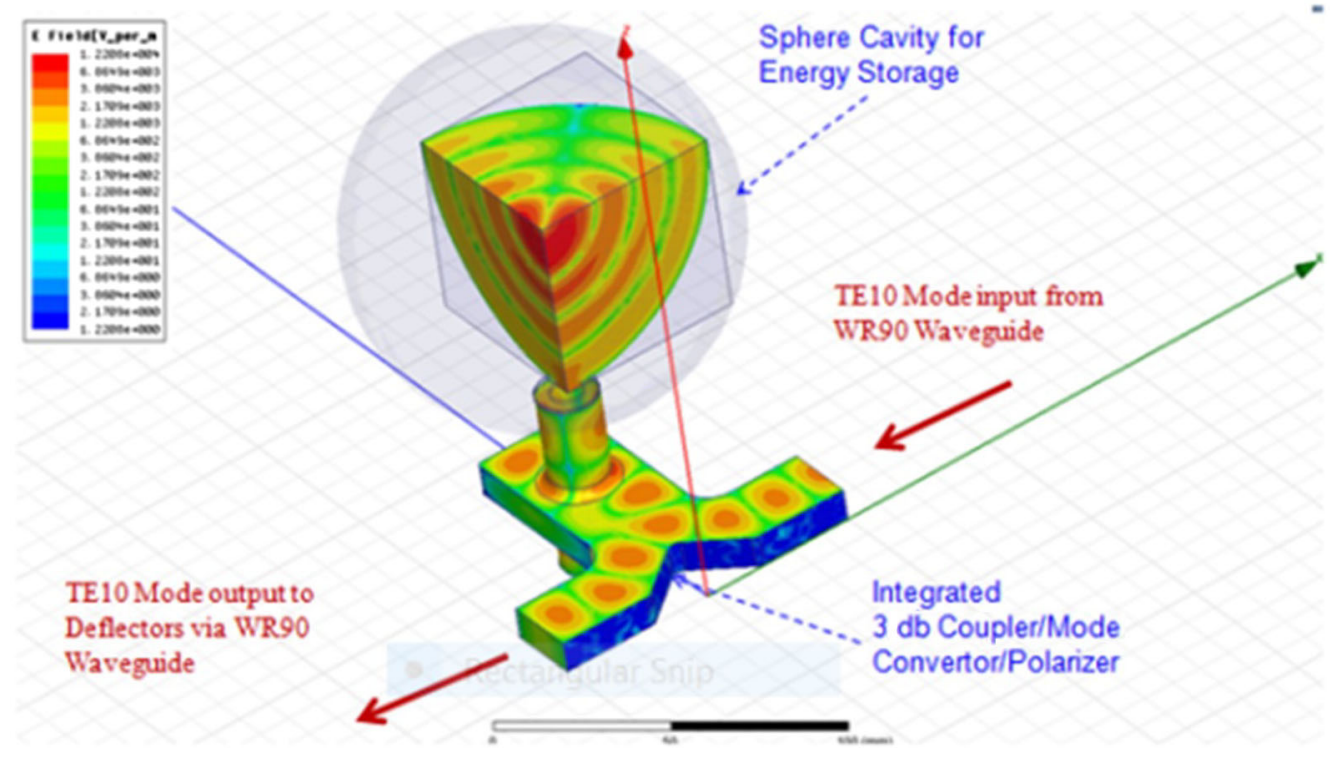

FIG. 8. The wave propagation in the SLED system.

types. All modes are concentrated at the cavity canter, away from the wall surface.

The quality factor $Q_{0}$ for TE modes is

$$
Q_{0}=\frac{a}{\delta}
$$

where $\delta$ is the skin depth $(0.61 \mu \mathrm{m}$ at $11 \mathrm{GHz}$ for copper at $20^{\circ} \mathrm{C}$ ).

Figure 7 shows two polarized $\mathrm{TE}_{114}$ SLED modes at $11424 \mathrm{MHz}$ as working SLED modes in a $\mathrm{Q}_{0} \sim 95000$ spherical cavity with an overcoupling $\beta$ coefficient of 7-8. A third $\mathrm{TE}_{014}$ mode with $7 \mathrm{MHz}$ higher frequency is shown on the left side in Fig. 7, which is very weakly undercoupled due to its field pattern. Figure 8 shows the wave propagation through the SLED system; both input and output ports are WR90 waveguides for the TE 10 mode. The

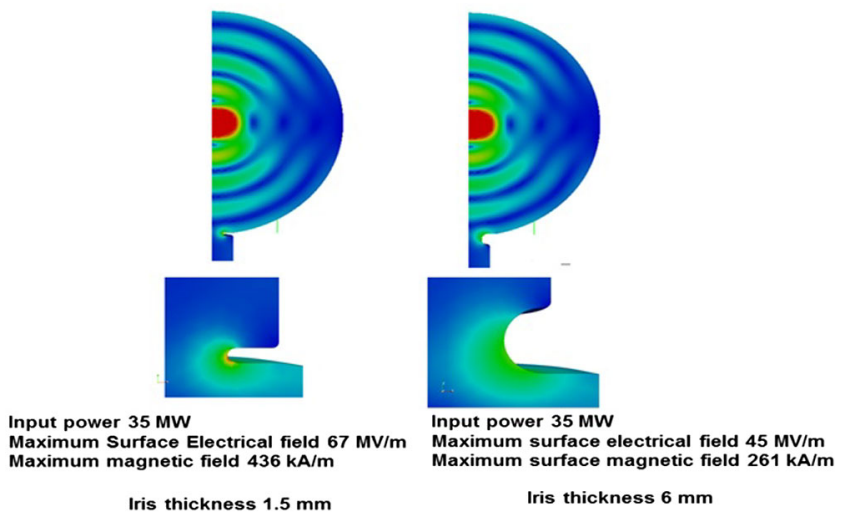

FIG. 9. Computer simulation of the field pattern (top) and maximum electric and magnetic field (bottom) for two types of coupling irises: iris thickness 1.5 (left) and $6 \mathrm{~mm}$ (right). transmission between these two ports is almost perfect with a negligible loss; the complete microwave measurement results will be discussed in a later section.

\section{Coupler design}

The coupling iris between the circular guide and sphere cavity was carefully studied and optimized to reduce the $\mathrm{rf}$ pulse heating. The final iris has a rounding radius of $3 \mathrm{~mm}$ to make the pulse heating less than $40^{\circ} \mathrm{C}$ for input $1.5 \mu \mathrm{s}$ square pulses at a peak power of $50 \mathrm{MW}$. Figure 9 shows the results of a computer simulation of the field patterns and amplitude for the couplers with two different iris radii (iris thickness 1.5 and $6 \mathrm{~mm}$, respectively).

\section{Mechanical design and assembly}

Figures 10 and 11 are pictures to show the fabricated polarizer and SLED assembly. In order to provide adequate pumping, a vacuum port was built in the polarizer bottom shown in the cutaway view in Fig. 11.

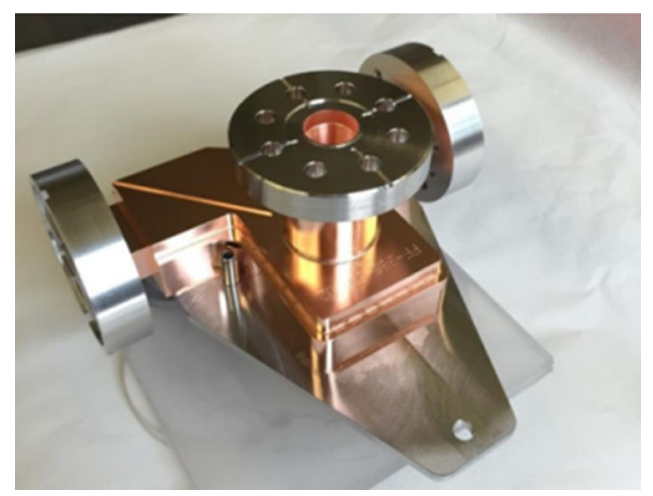

FIG. 10. A 3 dB coupler-mode convertor-polarizer. 

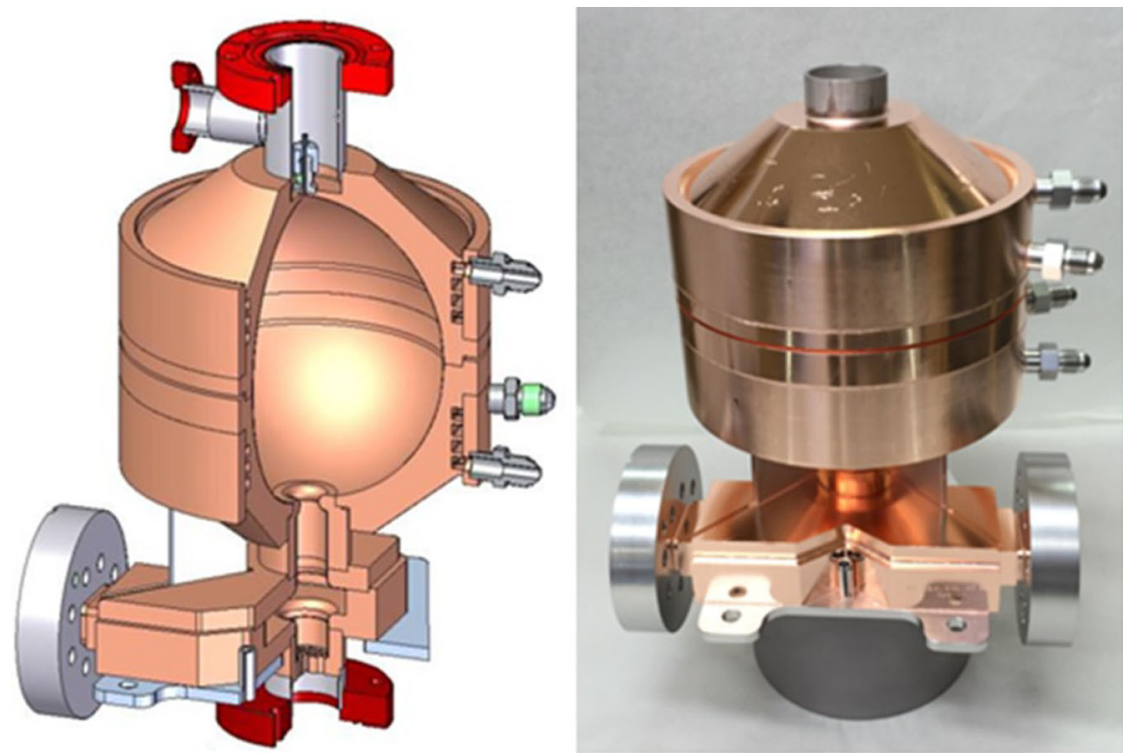

FIG. 11. Mechanical design and fabricated SLED assembly.

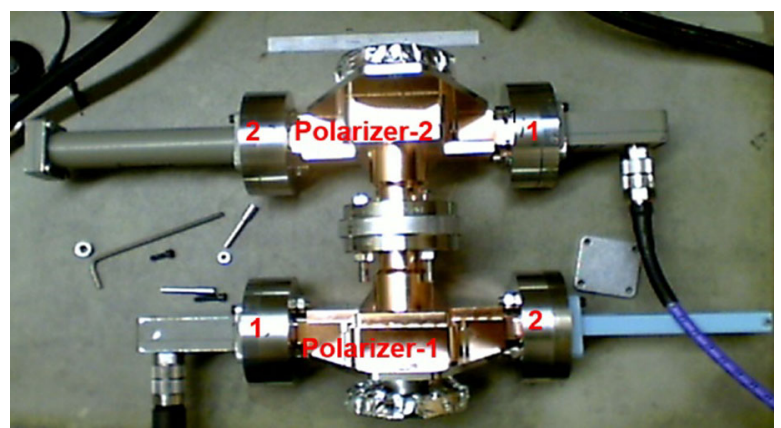

FIG. 12. Two polarizers are connected back to back with the circular waveguide ports.

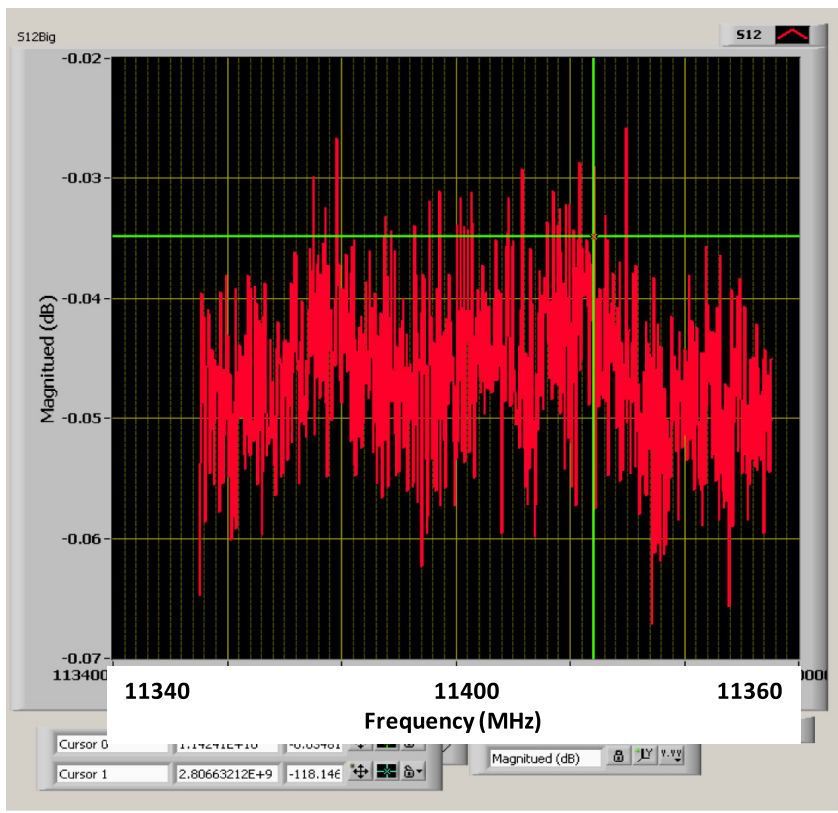

Tuning of the sphere cavity was well performed by a combination of machining away a very small amount of a tuning band at the sphere's equator and final tuning by a push-pull screw, which was brazed on the top region of the sphere. Because the design and machining were very accurate, only a tiny push was needed.

\section{MICROWAVE MEASUREMENTS}

\section{A. Perfect characteristics of polarizer}

When microwave measurements on the two brazed polarizer assemblies were performed, they both showed superior characteristics. Figure 12 shows the measurement

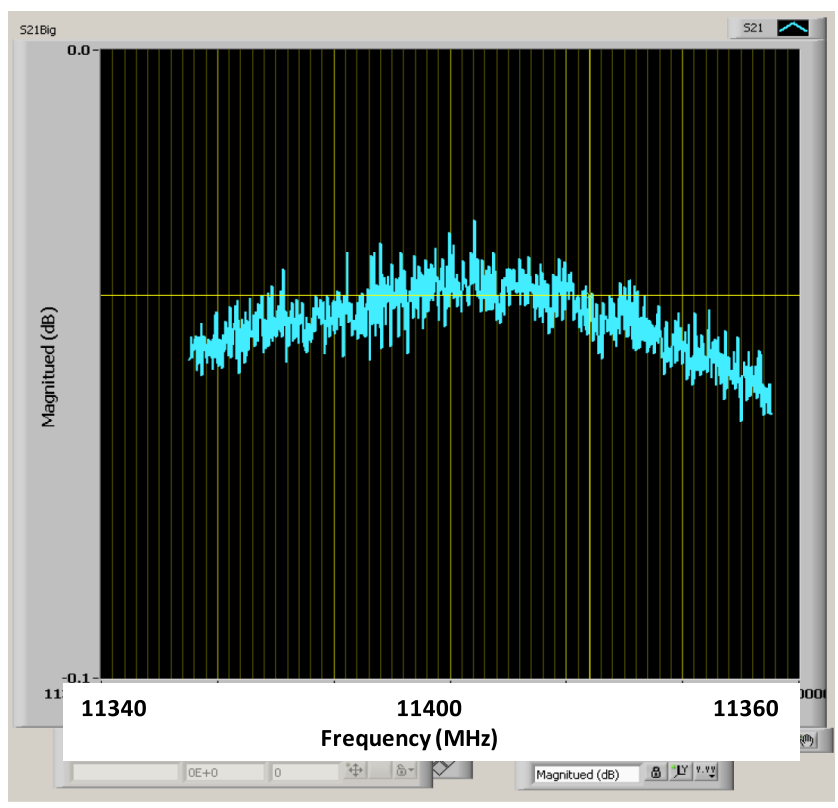

FIG. 13. Transmission is $-0.04 \mathrm{~dB}$ through two back-to-back polarizers. The transmission efficiency is $99 \%$. 

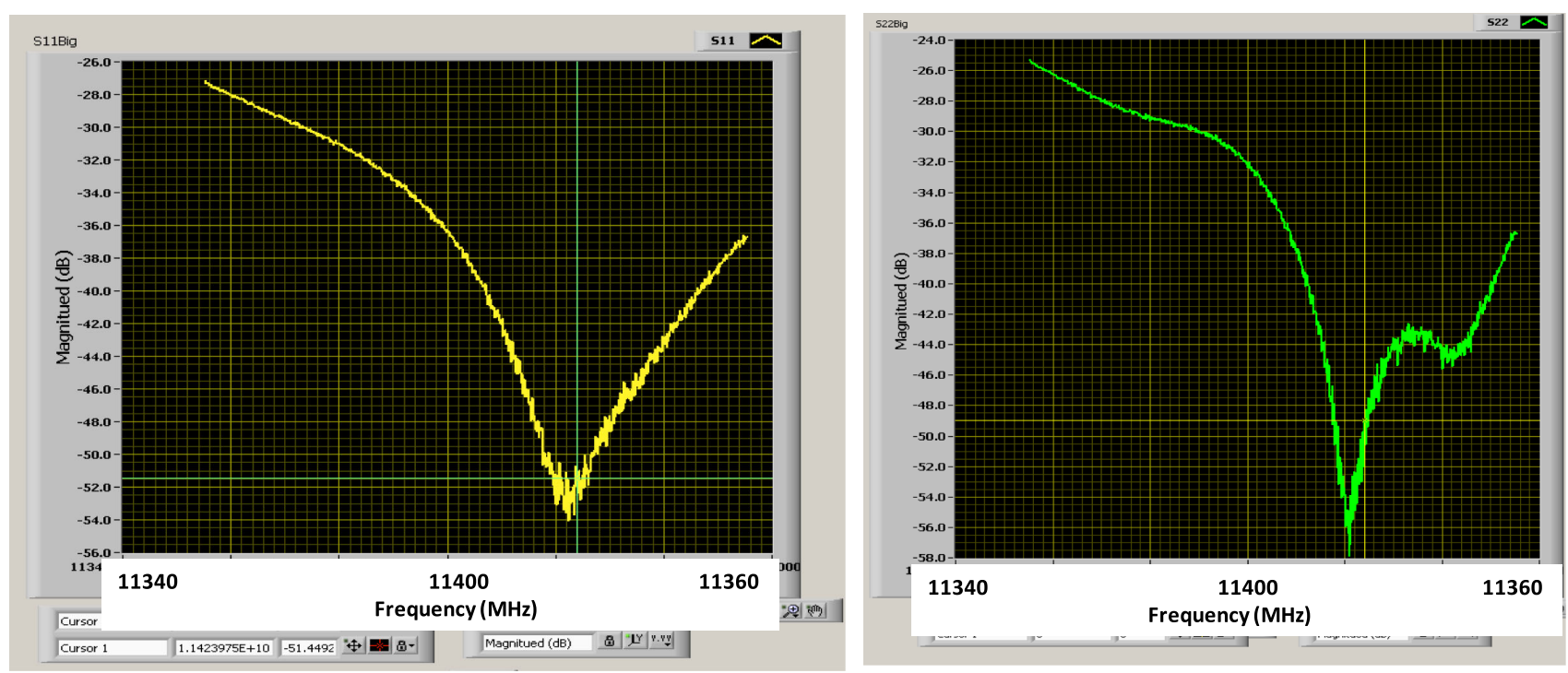

FIG. 14. Reflection from the input port is negligible $(-45 \mathrm{~dB})$.
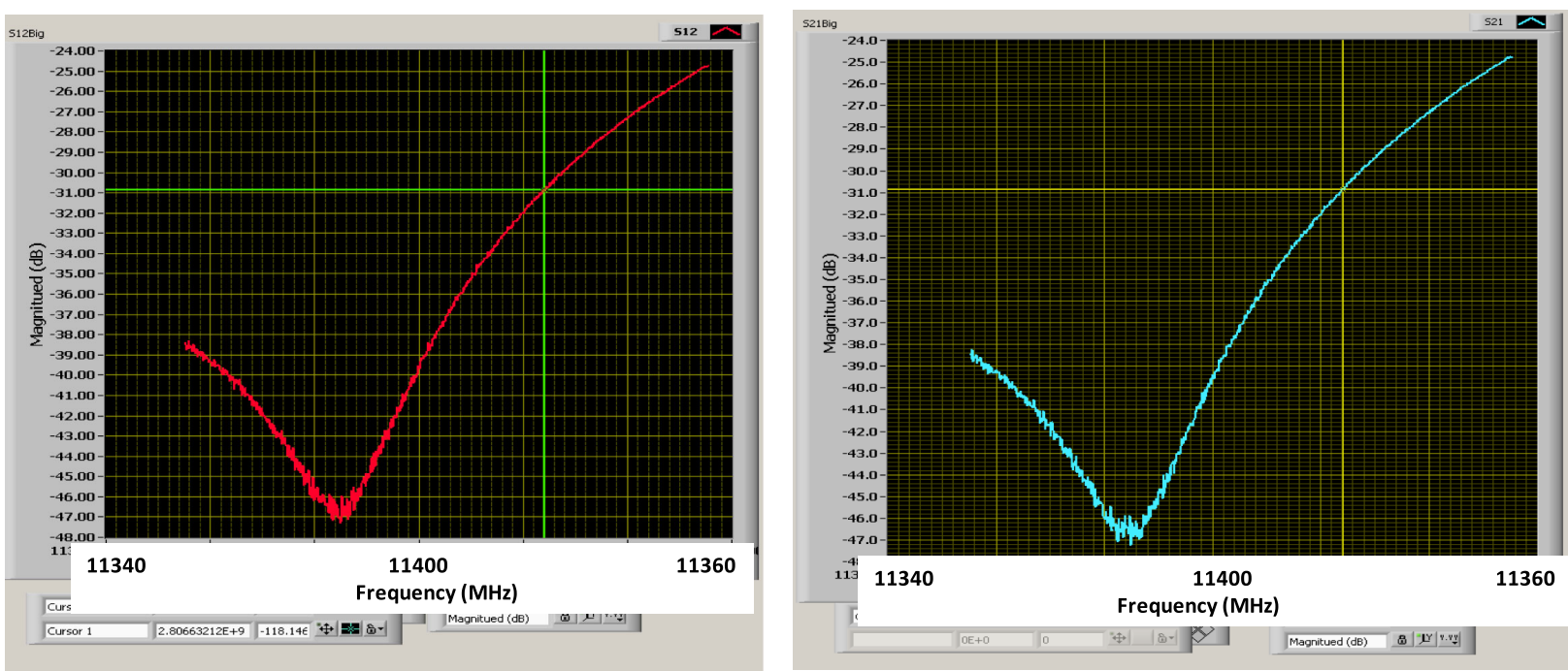

FIG. 15. Isolation between in and out WR90 ports is $-31 \mathrm{~dB}$.

setup; two polarizers were bolted together back to back through a spacer made for good electrical contact.

The following is a summary of the microwave measured properties: (i) The transmission is around $-0.04 \mathrm{~dB}$ for two back-to-back polarizers. It means the transmission efficiency is better than $99 \%$ as shown in Fig. 13. (ii) The reflection from the input port is around $-45 \mathrm{~dB}$. It means the reflection to the power source is negligible as shown in Fig. 14. (iii) The isolation of two WR90 ports is around $-31 \mathrm{~dB}$. It means the power source and deflector are completely isolated from each other as shown in Fig. 15. (iv) Broadband transmission over more than $100 \mathrm{MHz}$ with a center in $11424 \mathrm{MHz}$ means the polarizer can stably work with any change of the klystron working frequency as shown in Fig. 14.

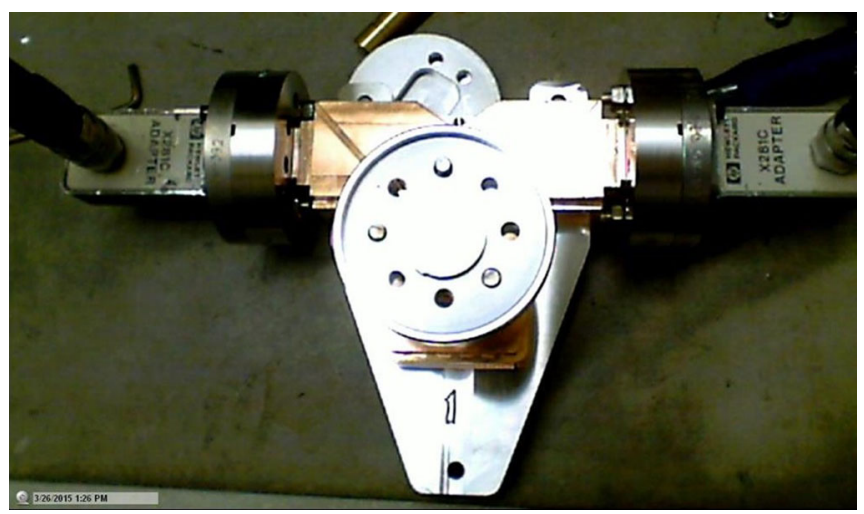

FIG. 16. Proof of full SLEDed power transmission with the end of circular waveguide shorted. 


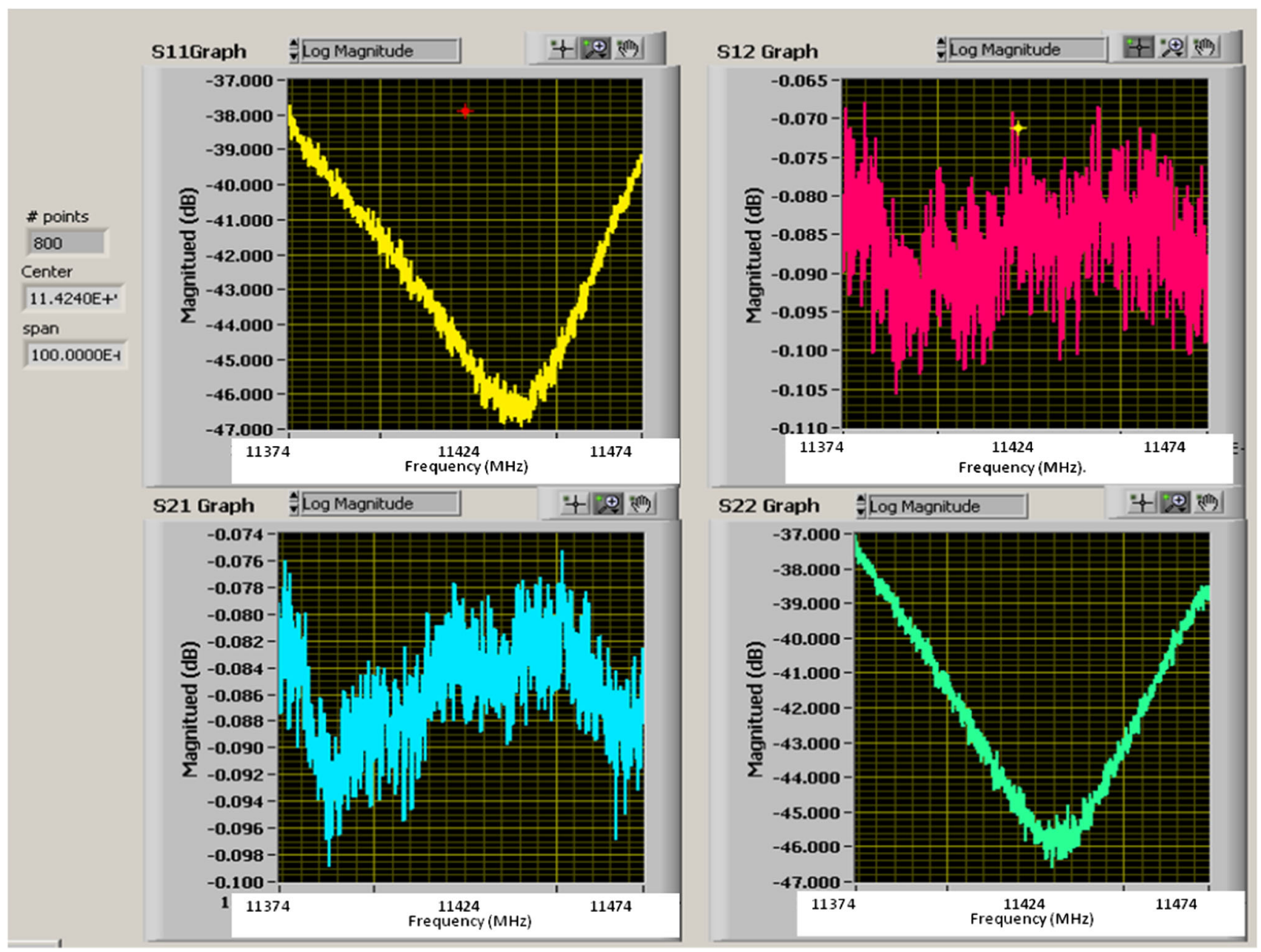

FIG. 17. Microwave measurement of full S parameters.

\section{B. Demonstration of SLED pulse transmission}

In order to examine the reflection property of the circular waveguide, we put a shorting plate in the circular port to allow full power reflected back as shown in Fig. 16.

Figure 17 shows all $\mathrm{S}$ parameters for the above measurement. The input port still has better than $-45 \mathrm{~dB}$ negligible reflection, and full power around $-0.05 \mathrm{~dB}$ was transmitted across to the other WR90 port. This is the exactly how the SLEDed pulse is supposed to work.

\section{System test}

After brazing the polarizer and spherical cavity together, the whole system was tested as shown in Fig. 18. The cold test has demonstrated excellent agreement between its microwave properties and the theoretical design. Figure 19 shows the two overlapped working modes $\mathrm{TE}_{114}$ and a $7 \mathrm{MHz}$ higher-frequency mode $\mathrm{TE}_{014}$, which is much undercoupled due to the orientation of this mode and sphere cavity shown in Figs. 19 and 20.

\section{HIGH-POWER OPERATION}

This new SLED system was high-power processed on an $X$-band station at the SLAC test lab and then was installed between an XL-4 klystron and upstream transmission line

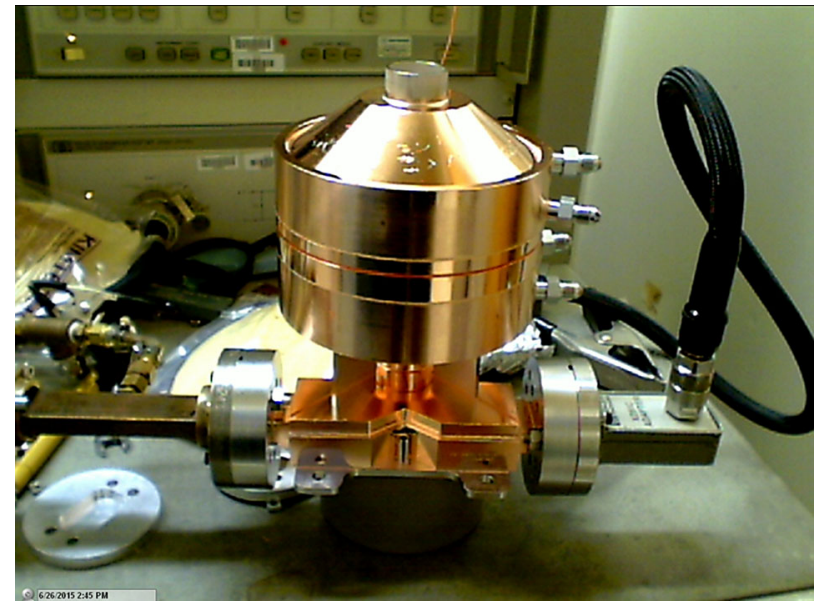

FIG. 18. Microwave measurement of full S parameters. 

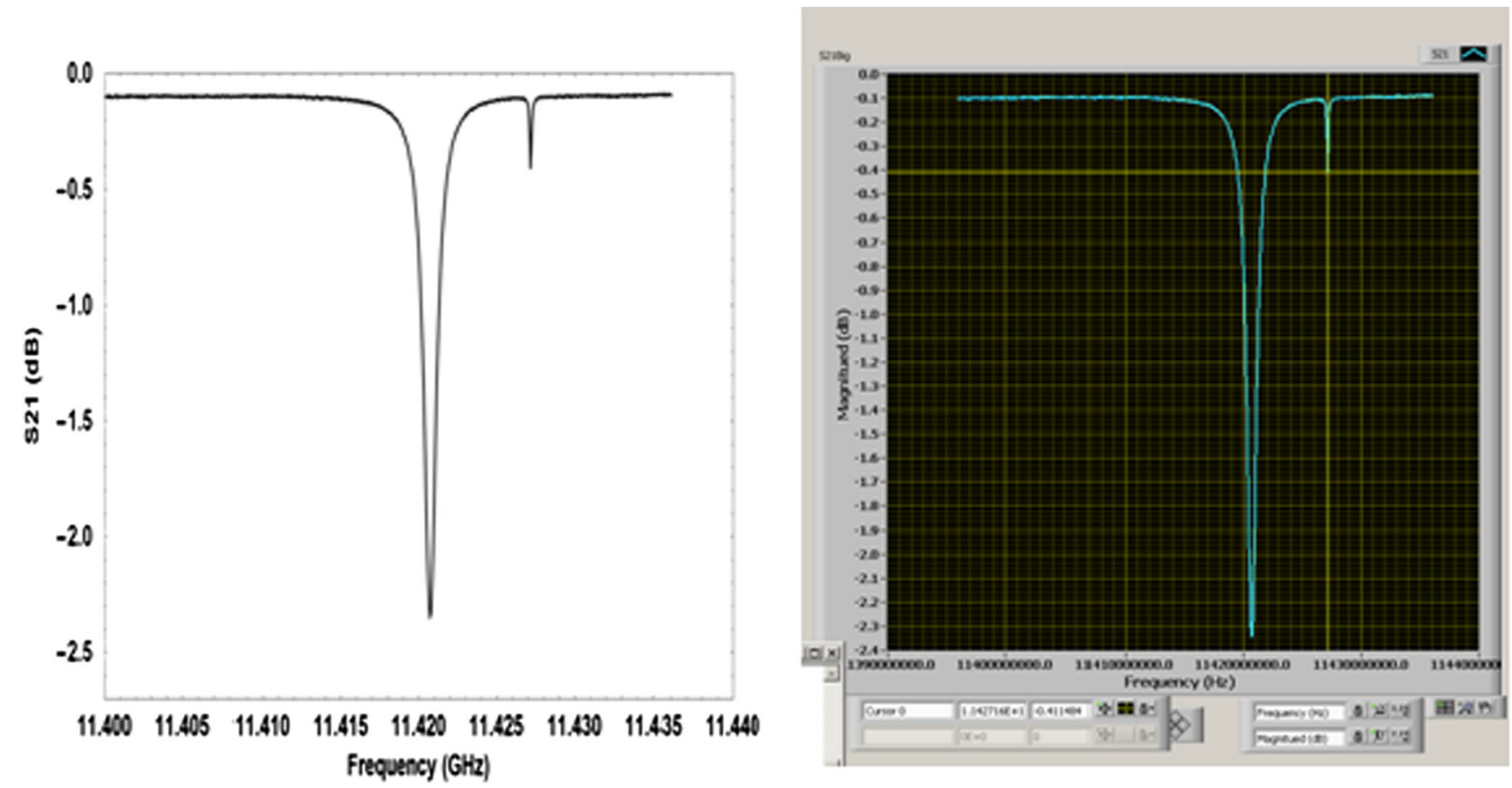

FIG. 19. Frequency scan showing the two overlapped working modes $\mathrm{TE}_{114}$ (large) and a $7 \mathrm{MHz}$ higher-frequency mode $\mathrm{TE}_{014}$ (small) for the theoretical design (left) and measurement (right).

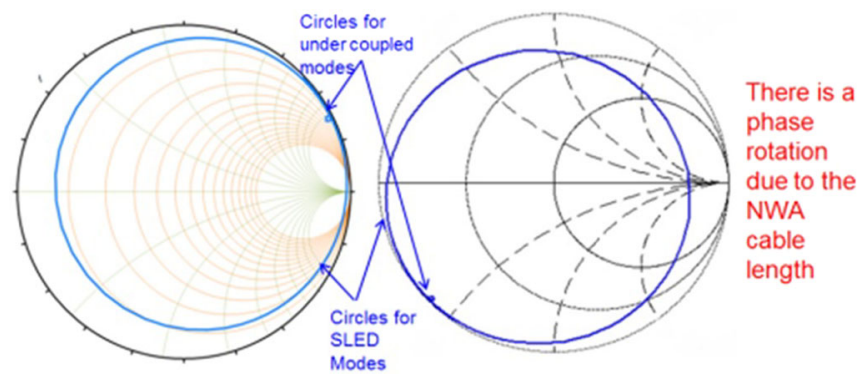

FIG. 20. Smith chart showing an overcoupled working mode (large circle) and undercoupled mode (tiny circle) for the theoretical design (left) and measurement (right).

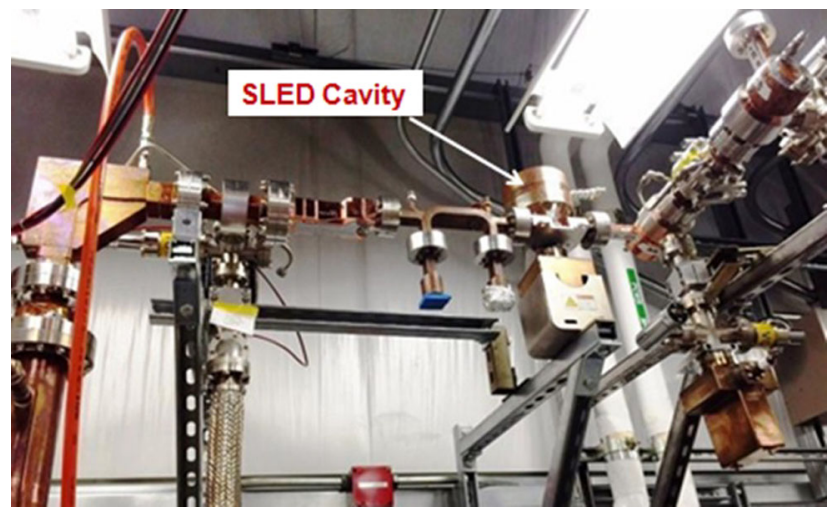

FIG. 21. The SLED system has been installed. for the deflectors in the support building above the beam line tunnel at the LCLS as shown in Fig. 21 [6]. The rf waveforms for the high-power operation in comparison with the theoretical calculation are shown in Fig. 22.

The maximum kick voltage of the two deflectors has increased from $45 \mathrm{MV}$ without SLED to $95 \mathrm{MV}$ during recent operation. The system was operated at a little lower than the maximum klystron power of $50 \mathrm{MW}$, because there was no practical need for the beam diagnostics. The whole system has been running encouragingly stable without breakdown, sign of pulse heating, outgassing, or rf contact radiation near the SLED cavity body.

The SLED cavity is cooled from a separate chiller system so that its temperature may be set independently to either tune or detune the system. When tuned, the SLED cavity is kept at $68^{\circ} \pm 0.5^{\circ} \mathrm{F}\left(20^{\circ} \pm 0.3^{\circ} \mathrm{C}\right)$ with a maximum compressed power level as shown in Fig. 23 [7]. The $3 \mathrm{~dB}$ frequency band of the SLED cavity is $(11424 \mathrm{MHz}) /(Q 0 /(1+\beta))=0.97 \mathrm{MHz}$. The corresponding temperature change range is about $5.1^{\circ} \mathrm{C}$ $\left(9.2^{\circ} \mathrm{F}\right)$. If it is desired to run the system in non-SLED mode, the temperature of the cavity can be raised by a nominal $20^{\circ} \mathrm{C}$. At the detuning temperature of $40^{\circ} \mathrm{C}$, the SLED is completely detuned. In the non-SLED operation, the fill length klystron pulses with $1.5 \mu$ s duration of up to 50 MW peak power can be applied to the deflecting structures. The chosen method of using the temperature to detune the sphere cavity avoids the use of expensive and complex mechanical tuners for the purpose. 

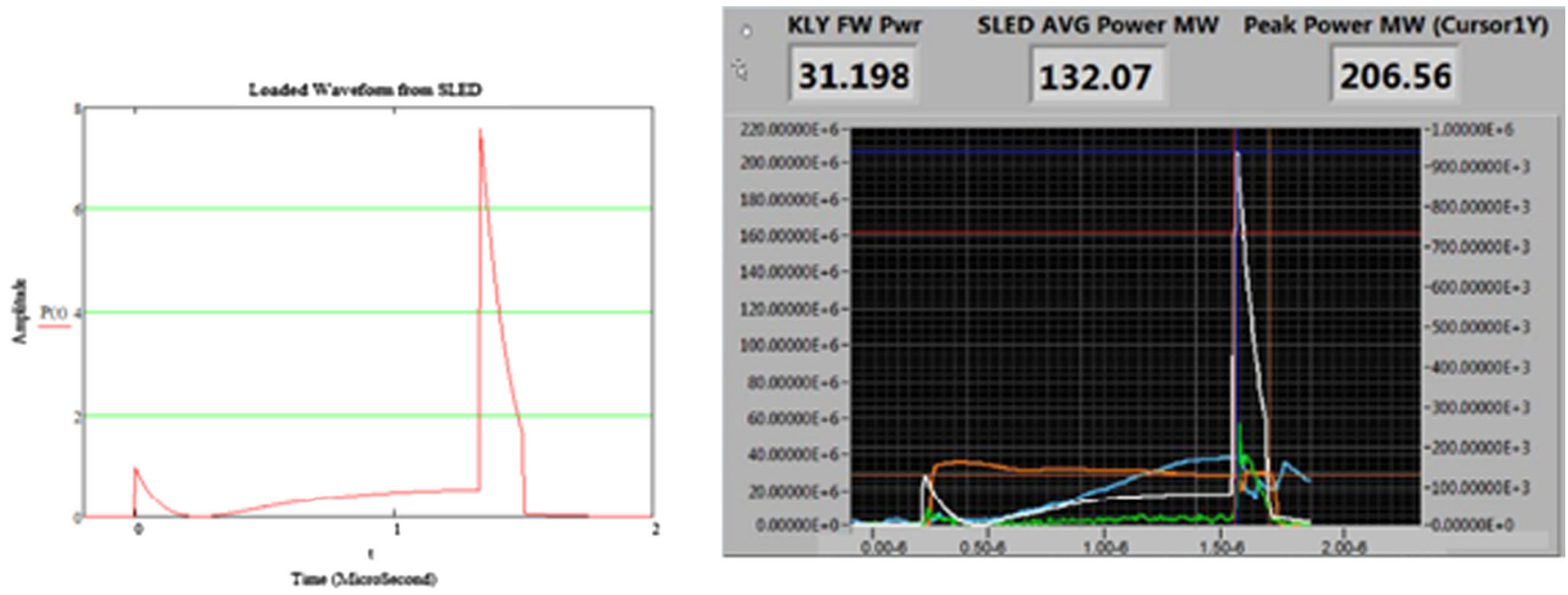

FIG. 22. High-power operation waveforms for the theoretical design (left) and measurement (right).

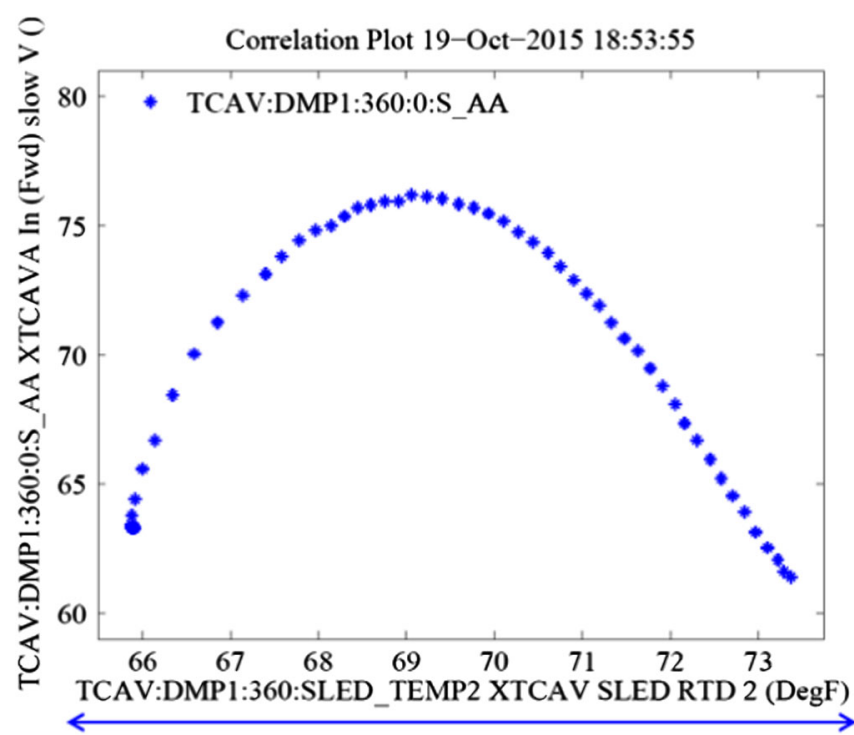

$3 \mathrm{~dB}$ Band Width $9.2^{\circ} \mathrm{F}$

FIG. 23. The temperature sensitivity of the SLED cavity is shown when the output power [MW] is plotted versus the temperature [degrees Fahrenheit].

\section{SUMMARY}

This progress report has proved great success for the $X$-band supercompact SLED system research and development.

Our design can be easily extended to C-band or S-band projects. A broad range of other future applications of the spherical energy storage cavity and innovative polarizer are on the horizon.
Possibilities for a flattop pulse compression system using pulse correction cavities are under extensive studies.

\section{ACKNOWLEDGMENTS}

This work was supported by Department of Energy Contract No. DE-AC03-76SD00515.

[1] C. Behrens, F.-J. Decker, Y. Ding, V. A. Dolgashev, J. Frisch, Z. Huang, P. Krejcik, H. Loos, A. Lutman, T. J. Maxwell, J. Turner, J. Wang, M.-H. Wang, J. Welch, and $\mathrm{J}$. Wu, Few-femtosecond time-resolved measurements of X-ray free-electron lasers, Nat. Commun. 5, 3762 (2014).

[2] J. W. Wang, S. G. Tantawi, and X. Chen, in Proceedings of the 27th International Linear Accelerator Conference (LINAC2014) (JACOW, Geneva, Switzerland, 2014).

[3] Z. D. Farkas, H. A. Hoag, G. A. Loew, and P. B. Wilson, Report No. SLAC-PUB-1453, 1974.

[4] M. Franzi, J. Wang, V. Dolgashev, and S. Tantawi, A compact rf polarizer and its application to pulse compression systems, Phys. Rev. Accel. Beams 19, 062002 (2016).

[5] R. F. Harrington, Time-Harmonic Electromagnetic Fields (McGraw-Hill, New York, 2001).

[6] J. W. Wang, S. G. Tantawi, C. Xu, M. Franzi, P. Krejcik, G. Bowden, S. Condamoor, Y. Ding, V. Dolgashev, J. Eichner, A. Haase, J. R. Lewandowski, and L. Xiao, in Proceedings of the 7th International Particle Accelerator Conference (IPAC2016) (JACOW, Busan, Korea, 2016).

[7] P. Krejcik, G. B. Bowden, S. Condamoor, Y. Ding, V. A. Dolgashev, J. P. Eichner, M. A. Franzi, A. A. Haase, J. R. Lewandowski, T. Maxwell, S. G. Tantawi, J. W. Wang, L. $\mathrm{Xiao}$, and $\mathrm{C}$. $\mathrm{Xu}$, in Proceedings of the 5th International Beam Instrumentation Conference (IBIC2016) (JACOW, Barcelona, 2016). 